\title{
Energy Harvesting Process Modelling of an Aeronautical Structural Health Monitoring System Using a Bond-Graph Approach
}

\author{
Thomas Sainthuile ${ }^{1, *}$, Sé bastien Gronde ${ }^{1}$, Chris tophe Delebarre ${ }^{1}$, Stéphane Godts ${ }^{2}$, Chris tophe Page t \\ ${ }^{1}$ IEMN-DOAE UMR CNRS 8520, University of Valenciennes and Hainaut-Cambrésis, Le Mont-Houy, Valenciennes, 59313, France \\ ${ }^{2}$ University of Valenciennes and Hainaut-Cambrésis, Le Mont-Houy, Valenciennes, 59313, France \\ ${ }^{3}$ AIRBUS OPERATIONS LIMITED, NDT \& Testing Technology, Filton, Bristol, BS99 7AR, UK
}

\begin{abstract}
Energy Harvesting is a promising solution for powering Structural Health Monitoring (SHM) systems since various mechanical energy sources are generated by aircraft. Today, the main technique to harvest energy consists of using a specific conversion device to provide power to the SHM system. In this paper however, a novel technique to obtain a self-powered SHM system for aeronautical structures is proposed. This SHM system aims to have a double functionality: it will carry out classical SHM tasks using piezoelectric transducers bonded onto the aircraft structure and will also be fully autonomous since the same transducers will convert the mechanical vibrations of the structure into electrical power. Using a bonded piezoelectric transducer to harvest energy will also bring wideband frequency energy harvesting capability. This autonomous system using a unique transducer being particularly innovative, the objective of this paper is to provide a complete Bond Graph model of the energy harvesting process in order to allow the optimisation of its performances. This approach is well-suited to monitor the power and energy transfer carried out during the process since it takes into account the interaction between multiphysics systems, here the electrical and mechanical domains in terms of power and energy variables. Consequently, each part of the energy harvesting, i.e. the mechanical vibration of the host structure, the vibration within the SHM energy harvester volume, the piezoelectric electromechanical conversion and the terminal electric load have been modelled analytically using this Bond Graph approach. Then, each submodel has been verified with a baseline Finite Element model. Good agreements have been found and it has been possible to carry out an estimation of the power harvested by the SHM energy harvester for a given mechanical excitation using this innovative complete analytical Bond Graph model.
\end{abstract}

Keywords SHM, Energy Harvesting, FEM, Mechanical Vibrations, Analytical Modelling, Bond Graph, Power Estimation, Aeronautical Structures

\section{Introduction}

Nowadays, Structural Health Monitoring (SHM) systems have become a part of new avionic systems which will form future aircraft. Their objectives are to detect and locate damages occurring within the aircraft structure. They also have to be able to characterize the nature and the gravity of the damage as well as to estimate the remain ing lifespan of the structure. The place of such systems is rapidly evolving. They are currently used duringdevelopment test campaigns. However, a technological gap must be overtaken as a major objective is to use them on production line and also to implement them on-board aircraft.

* Corresponding author:

thomas.sainthuile@univ-valenciennes. fr (Thomas Sainthuile)

Published online at http://journal.sapub.org/aerospace

Copyright (C) 2012 Scientific \& Academic Publishing. All Rights Reserved
To ensure a good efficiency of the SHM system, a sensor network covering the structure has to be designed. These SHM transducers will have to be integrated and/or spread out over large areas like wings and fuselage to monitor the health of the structure. Integrating these transducers within the aircraft in hard access areas implies the need of wireless communication capabilities as well as energy autonomy to minimize the wire clutter.The autonomy capability is also required to avoid the use of batteries which have to be re-placed over the time and cause is sues with their recycling process. Moreover, with the trend to the all-electric aircraft, more and more equipments need power. That is why the energy management of SHM devices is a very strategic topic as they must be designed as autonomous systems. Several energy harvesting solutions are available and many authors investigated their efficiencies with growing interests in the last few years. Mathuna et al.[1] and Harb[2] proposed a synthesis of the energy harvesting solutions. In[3], Park et al. particularly focused on the energy harvestingsolutions which 
can be used in SHM systems. This work provided the trend for further studies. The conversion from mechanical vibration into electrical energy, the thermal gradient and the Radio Frequency (RF) energy conversion have been more particularly investigated subsequently. Samson et al.[4] presented a system based on the thermal conversion using an independent energy source comb ined with a wireless sensor unit. In[5], Roundy et al. investigated the potential of electro-magnetic, electrostatic and piezoelectric vibration conversion. Following this work, Roundy et al.[6] continued to study the voltage response of piezoelectric cantilever beams used as energy harvester. Many other contributions can be found on the study of piezoelectric cantilever beams. One can cite the works from Lefeuvre et a1.[7] and Lallart et al.[8-9] who more specifically focused on the optimization of the loading circu it using non-linear techniques to increase the power harvested level. Farinholt et a1.[10] proposed an autonomous SHM system for civilian structures harvesting energy from multiple environment sources, or periodically delivered by a RF energy source. In[11], Ferrari et al. used several piezoelectric cantilever beams on which different masses have been bonded to convert energy over an extended frequency range. All these prior works demonstrated the possibility of using cantilever beams to carry out energy harvesting with a level of power harvested ranging from the hundreds of microwatt to the milliwatt. These results make this technique a particularly promising candidate for energy harvesting.Another contribution of these works is that a complete analytical response can be derived from the natural vibration of the beam.

Here, the technology retained by the authors for the energy harvesting is not based on cantilever beams. The overall a im of their technology is to build an innovative SHM system having a double functionality. This system has to be able to carry out, only with the piezoelectric transducers bonded onto the structure, the classical SHM tasks like damage detection and/or location, and also harvest energy to be fully autonomous. This system, compared to cantilever beams, will not be centered around a single frequency but will work over an extended frequency range. Moreover, having a unique transducer for SHM and energy harvesting purposes reduces the number of devices required for the system. This is particularly interesting when, on-board aircraft, one tends to implement systems in hard access areas with wireless connection. According to these requirements and to the previous literature, the mechanical vibration conversion seems to be an appropriate technology. Sodano et al.[12] presented such a configuration, showing the possibility of harvesting energy with a bonded piezoelectric sensor. In[13-15], the authors presented their SHM autonomous system and checked the feasibility of harvesting energy with the piezoelectric SHM energy harvester.

However, the configuration of a SHM energy harvester bonded onto the vibrating structure differs drastically compared to the use of a piezoe lectric cantilever beam and an analytical model has to be created to obtain a fundamental understanding of the piezoelectric energy harvesting process.
To do so, using the Thevenin equivalent circuit, the piezoelectric energy harvester is represented as a voltage source connected in serial with its internal output impedance $\mathrm{Z}$, as shown in Figure 1. With this representation, and to evaluate its power harvesting capabilities, one has to determine both its voltage response and the current delivered to the load.

Several models of the voltage response of bonded transducers have been developed. Prior works have been proposed by Di Scalea et al.[16], Raghavan et al.[17] and Chapuis[18], who have used a plane-stress model to obtain the voltage response of a thin piezoelectric transducer subjected to a Lamb wave excitation. However, the study of the power transferred to a load by the piezoelectric transducer was beyond the scope of these articles and consequently not carried out.

In this paper, an innovative model of the complete energy harvesting process will be proposed using the Bond Graph approach to carry out the evaluation of the energy harvesting capabilities of the SHM energy harvester as shown in Figure 1. It will allow the determination of the power harvested by a SHM energy harvester due to mechanical vibrations since the Bond Graph technique is well-suited to study the power exchanges in multiphysics systems, i.e. between the mechanical and the electrical domains[19, 20]. In this representation, the inputs and outputs of each subsystem are defined by power variables represented by a conjugated pair of effort / flow variables i.e. the pair $F, v$ for the mechanical domain (force and velocity) and $V, i$ for the electrical doma in (voltage and current).

The configuration retained herein consists of a set of piezoelectric SHM energy harvesters bonded onto an aluminium plate simply supported on its four edges and associated with a terminal electric load. The SHM energy harvesters were primarily used to monitor the health of the structure with classical SHM techniques such as the electromechanical impedance technique[21], the Selective Lamb Mode Technique[22], Acoustic Emission Monitoring $[23,24]$ and Lamb waves interaction $[25,26]$. In this study, the structure is excited by an electro mechanical shaker at its resonant frequencies. When vibrating at these resonant frequencies, the structure provides displacements to the bonded energy harvesters which exhibit a voltage and subsequently provide power to the terminal electrical load.

For each subsystem of the Bond Graph model, shown in Figure 1, representing each step of the energy harvesting process, the phenomena will be analytically described to allow the determination of the required data. The subscript $i$ refers to the three space directions. The superscript $\mathrm{P}$ refers to the Pie zoelectric energy harvester while the superscript $S$ stands for the Structure.

First, the piezoelectric electro-mechanical conversion will be presented in section 2 . This subsystem has for input the mechanical force $F_{i}^{P}$ and velocities $v_{i}^{P}$ applied to the energy harvester. They are multibond graph elements as they take into account the three space directions. It has for outputs the voltage $V^{P}$ and current $i^{P}$ due to the electro mechanical 
conversion. Then, the structural mechanical vibrations will be treated in section 3 . The displacements of the plate due to the external mechanical excitation noted $F^{\text {shaker }}$ will be determined. $F^{\text {shaker }}$ is the input signal of the block diagram representing the energy harvesting process. These displacements will allow the determination of the velocities $v_{i}^{P}$ applied to the SHM energy harvester. The modelling of the terminal electric load will be carried out in section 4 . The output $i^{L}$ and $V^{L}$ will lead to the power harvested and transferred to a resistive load. In section 5, the results of the simulation using this model will be presented. A Finite Element Method (FEM) model will be used to validate and discuss the accuracy of the model.

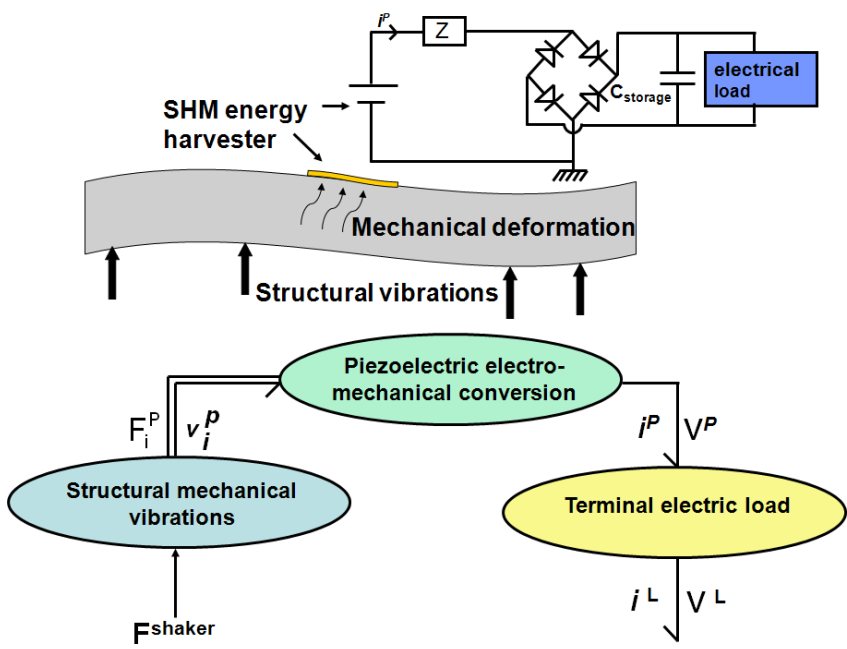

Figure 1. Energy harvesting process and associated Word Bond Graph

\section{PiezoelectricElectro-Mechanical Conversion}

In this part, the electro-mechanical conversion carried out by the SHM energy harvester is studied. This subsystem is the key part of the global energy harvesting system since it depends on the mechanical excitation from the structure it is bonded onto, and also provides the harvested power to a terminal electric load.

The analytical voltage response of the SHM energy harvester is obtained by coupling the direct piezoelectric effect to the strain field applied to the energy harvester. The major feature of the analytical model is its ability to provide an easily computable voltage response for any kind of deformation. Although Di Scalea et al.[16], Raghavan and Cesnik[17] and Chapuis[18] only considered plane stress case for the transducer response to mechanical vibration, Ali[27,28] developed a more accurate model taking into account the stress distribution throughout the thickness of the transducer. Here, a three dimensional (3D) model of the transducer response, i.e. taking into account the three space directions is proposed. The piezoelectric SHM transducers used herein are PZT (Lead Zirconate Titanate) Pz 27 from FerropermPiezoceramics $\mathrm{S} / \mathrm{A}$, i.e. having a $6 \mathrm{~mm}$ hexagonal crystal system.
Using the PZT transducer as a SHM energy harvester, the following set of equations is more appropriate compared to the other sets of piezoelectric constitutive equations ([18]).

$$
\begin{gathered}
T_{i j}^{P}=-h_{k i j} D_{k}^{P}+C_{i j k l}^{D} S_{k l}^{P} \\
E_{i}^{P}=-h_{k i j} S_{k l}^{P}+\beta_{i j}^{S} D_{j}^{P}
\end{gathered}
$$

with $\mathrm{i}, \mathrm{j}, \mathrm{k}, 1 \in[1,3]$.

$\mathrm{E}^{\mathrm{P}}$ is the electric field, $\mathrm{D}^{\mathrm{P}}$ the electrical displacement, $\mathrm{T}^{\mathrm{P}}$ the mechanical stress applied to the piezoelectric energy harvester, $S^{P}$ the mechanical strain, $h$ the piezoelectric matrix, $C^{D}$ the stiffness matrix measured with open circuit and $\beta^{S}$ the impermittivity matrix at constant strains. All these variables are listed in the Nomenclature.

For the piezoelectric SHM energy harvester considered in this study, electric charges are only present on the electrodes. Moreover, only the electric field and electrical displacement components parallel to the polarization direction a re non-null i.e. $D_{1}^{P}=D_{2}^{P}=0$ and $E_{1}^{P}=E_{2}^{P}=0$. As its voltage response would be measured using a high impedance device, one can consider the energy harvester being in open circuit conditions [16-18], i.e.:

$$
\iint D_{3}^{P} d x d y=0
$$

One also has

$$
\overrightarrow{E^{P}}=-\overrightarrow{\operatorname{grad}}\left(V^{P}\right)
$$

with $V^{P}$ the voltage exhibited by the SHM energy harvester.

The mechanical strain being defined as follows:

$$
S_{i j}^{P}=\frac{1}{2}\left(\frac{d u_{i}^{P}}{d x_{j}}+\frac{d u_{j}^{P}}{d x_{i}}\right)
$$

the voltage response can be written as:

$$
V^{P}=\frac{1}{\Gamma^{P}} \iiint_{\Omega^{P}}\left[h_{31}\left(S_{11}^{P}+S_{22}^{P}\right)+h_{33} S_{33}^{P}\right] d \Omega^{P}
$$

$\Omega^{\mathrm{P}}$ and $\Gamma^{\mathrm{P}}$ are the volume and the surface of the piezoelectric SHM energy harvester.

The current flowed into the piezoelectric static capacitance is derived from equation (5) as shown in equation (6).

with:

$$
i^{P}=C_{S} \frac{\partial V^{P}}{\partial t}=r_{1} v_{1}^{P}+r_{2} v_{2}^{P}+r_{3} v_{3}^{P}
$$

$$
\begin{gathered}
v_{i}^{P}=\frac{\partial}{\partial t} \iiint_{\Omega^{P}} \frac{1}{\Gamma^{P}} S_{i i}^{P} d \Omega^{P} \text { for } 1 \leq i \leq 3 \\
r_{1}=r_{2}=h_{31} C_{S} \\
r_{3}=h_{33} C_{S} \\
C_{S}=\epsilon_{33} \frac{L_{P} W_{P}}{T_{P}}
\end{gathered}
$$

LP, WP, Tp, $\varepsilon_{33}^{\mathrm{T}}$ correspond to the length, width, thickness and the permittivity coefficient at constant stress of the piezoelectric transducer, respectively. $v_{1}^{P}, v_{2}^{P}$ and $v_{3}^{P}$ correspond to the velocities of the transducer along its length, width and thickness, respectively.

The Bond Graph model of the piezoelectric electro-mechanical conversion is derived from equations (5) and (6) to (10) and is presented Figure 2. The three space directions are taken into account with the three branches corresponding to $\mathrm{v}_{1}^{\mathrm{P}}, \mathrm{v}_{2}^{\mathrm{P}}$ and $\mathrm{v}_{3}^{\mathrm{P}}$. The piezoelectric transformation is modelled by the TF elements $r_{1}, r_{2}$ and $r_{3}$. The current is the sum of the three contributions at the 0 -junction. The static capacitance of the energy harvester is added using the storage $\mathrm{C}$-element.

The submodel referred as the "Piezoelectric 
electro-mechanical conversion" is now completed and provides the voltage response $\mathrm{V}^{\mathrm{P}}$ of the SHM energy harvester in response to the strain field applied. Consequently, one has to obtain the required inputs coming from the structural mechanical vibrations and applied to the SHM energy harvester, i.e. $S_{11}^{\mathrm{P}}, S_{22}^{\mathrm{P}}$ and $S_{33}^{\mathrm{P}}$. The determination of these values is presented in the next section.

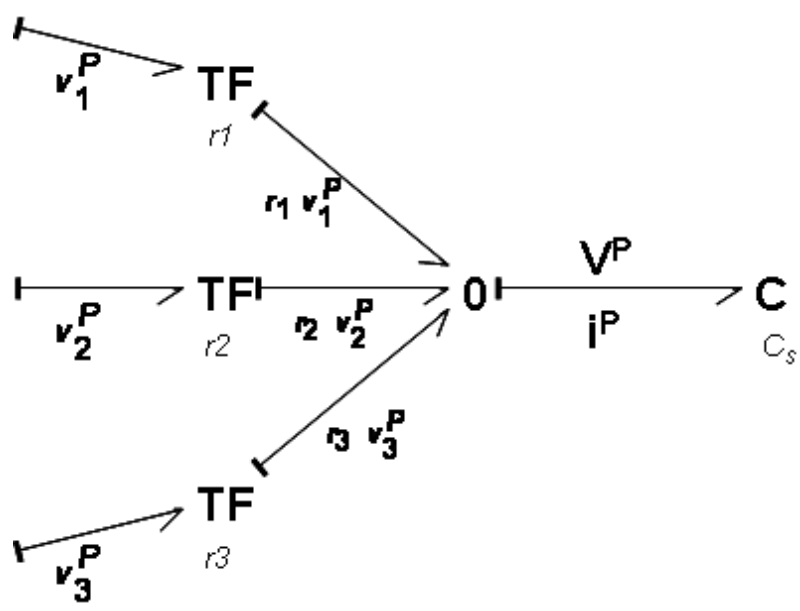

Figure 2. Bond Graph model of the SHM energy harvester

\section{Structural Mechanical Vibrations}

Figure 3 is a Bond Graph representation of equations (6) to (10). The input is the signal corresponding to the mechanical vibration $\mathrm{F}^{\text {shaker }}$. The strain field experienced by the energy harvester, $S_{11}^{\mathrm{P}}, S_{22}^{\mathrm{P}}, S_{33}^{\mathrm{P}}$ and subsequently the velocities $\mathrm{v}_{1}^{\mathrm{P}}$, $v_{2}^{\mathrm{P}}, \mathrm{v}_{3}^{\mathrm{P}}$ are determined analytically. The Bond Graph MSf elements are Modulated Sources Flow which are the output of the Structural Mechanical Vibrations submodel. They represent the harmonic excitation which is applied to the SHM energy harvester in the three space directions. $u_{i}^{S}$ are the displacement components at the top surface of the alu min iu m p late while $u_{i}^{\mathrm{P}}$ are the displacements components within the energy harvester. Knowing these parameters leads to the voltage response as shown in section 2.

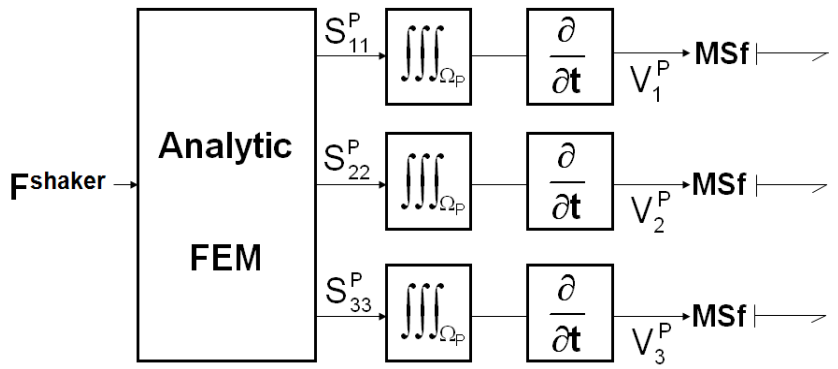

Figure 3. Bond Graph representation of the mechanical vibrations

Figure 4 presents the setup of the SHM energy harvester bonded onto the plate and Figure 5 presents the different displacement fields of the plate and of the SHM energy harvester. The length, width and thickness of the plate are noted $\mathrm{L}_{\mathrm{S}}, \mathrm{W}_{\mathrm{S}}, \mathrm{T}_{\mathrm{S}}$, respectively. A Cartesian basis, noted $\left(\mathrm{o}, \mathrm{x}_{1}, \mathrm{x}_{2}, \mathrm{x}_{3}\right)$ is defined as shown in Figure 5.

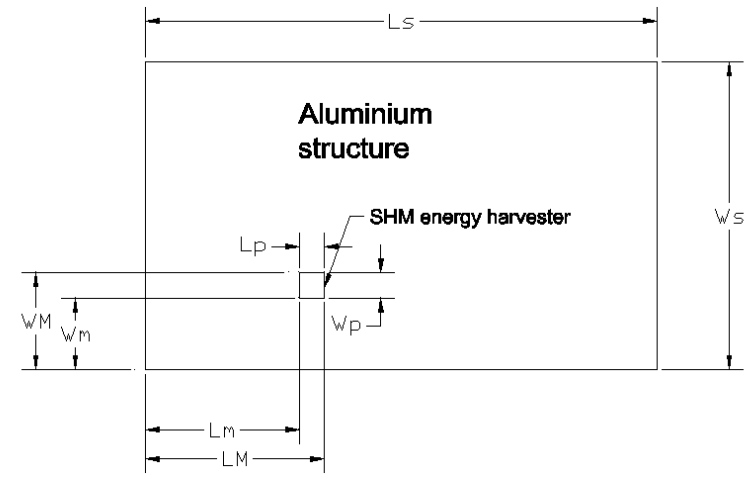

Figure 4. Dimensions of the structure and of the SHM energy harvester

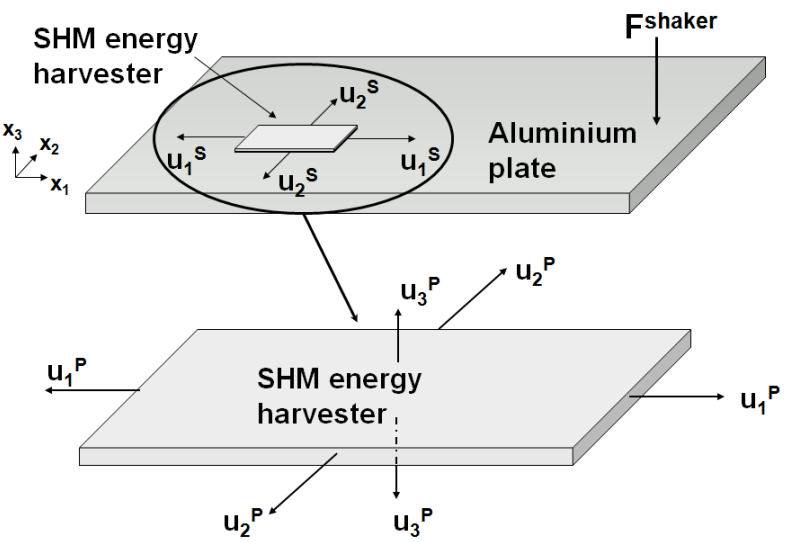

Figure 5. Displacements transmitted by the plate to the SHM energy harvester

To start with, it is necessary to model the vibrations occurring within the aluminium plate. This modelling is carried out using the plate theory.

The natural modes of a plate subjected to a mechanical punctual force applied by the shaker at $x_{1,0}, x_{2,0}$ coordinates can be determined analytically using the bending plate equation:

$$
\begin{gathered}
\rho T_{S} \frac{\partial^{2} u_{3}^{S}\left(x_{1}, x_{2}, t\right)}{\partial t^{2}}+D \nabla^{4} u_{3}^{S}\left(x_{1}, x_{2}, t\right)= \\
F^{\text {shaker }}(t) \delta\left(x_{1}-x_{1,0}\right) \delta\left(x_{2}-x_{2,0}\right)
\end{gathered}
$$

with $\rho$ the density of the material, $T_{S}$ the thickness of the plate, $D$ the bending stiffness, $E$ the Young modulus, $v$ the Poisson coefficient, $\nabla^{4}=\Delta^{2}$, the double Laplacian operator and $\delta$ the Dirac function. These operators are presented in the Nomenclature.

The total equation of displacement of a plate simply supported on its four edges is determined analytically as presented in[28]:

$$
u_{3}^{S}\left(x_{1}, x_{2}, t\right)=\sum_{m}^{\infty} \sum_{n}^{\infty} a_{m n}(t) \phi_{m n}\left(x_{1}, x_{2}\right)
$$

with $\phi_{m n}$ the modal deformation:

$$
\phi_{m n}\left(x_{1}, x_{2}\right)=\sin \left(\frac{m \pi}{L_{S}} x_{1}\right) \sin \left(\frac{n \pi}{W_{S}} x_{2}\right)
$$

With equations (12) and (13), the vertical component of displacement is completely determined analytically. The variable $a_{m n}(t)$ corresponds to the amplitude of displacement for the mode $(m, n)$ considered.Taking into account the mechanical damping $\xi$, the modal deco mposition presented in equation (14) provides the vertical displacement 
$u_{3}^{S}$ in response to an harmonic excitation $F_{m n}^{\text {shaker }} e^{j \omega t}$ as shown in equation (15).

$a_{m n}^{. *}(t)+2 \xi_{m n} \omega_{m n} a_{m n}^{\cdot}(t)+\omega_{m n}^{2} a_{m n}(t)=\frac{F_{m n}^{\text {shaker }}(t)}{M_{m n}}(14)$ with $\omega_{\mathrm{m}}$ the natural pulsation of the mode $(\mathrm{m}, \mathrm{n})$ considered, $\xi_{\mathrm{mn}}$ the damping factor, $\mathrm{M}_{\mathrm{mn}}$ the generalized mass, $\mathrm{F}_{\mathrm{mn}}^{\text {shaker }}$ the modal force. These variables are also presented in the Nomenclature.

$$
\left.u_{3}^{S}\left(x_{1}, x_{2}, t\right)\right|_{\omega}=\sum_{m}^{\infty} \sum_{n}^{\infty} \frac{F_{m n}^{\text {shaker }}}{M_{m n}\left[\omega_{m n}^{2}-\omega^{2}+j 2 \omega \omega_{m n} \xi_{m n}\right]}
$$

Using the Kirchhoff-Love plate theory, the in-plane displacements at the surface of the plate are derived from $\left.\mathrm{u}_{3}^{\mathrm{S}}\right|_{\omega}$. For a specific natural mode $(\mathrm{m}, \mathrm{n})$ one has:

$$
\begin{aligned}
& \left.u_{1}^{S}\left(x_{1}, x_{2}, t\right)\right|_{\omega_{m n}}=A_{1} \frac{\partial \phi\left(x_{1}, x_{2}\right)}{\partial x_{1}} e^{j \omega_{m n} t} \\
& \left.u_{2}^{S}\left(x_{1}, x_{2}, t\right)\right|_{\omega_{m n}}=A_{2} \frac{\partial \phi\left(x_{1}, x_{2}\right)}{\partial x_{2}} e^{j \omega_{m n} t} \\
& \left.u_{3}^{S}\left(x_{1}, x_{2}, t\right)\right|_{\omega_{m n}}=A_{3} \phi\left(x_{1}, x_{2}\right) e^{j \omega_{m n} t}
\end{aligned}
$$

with

$$
\begin{gathered}
A_{1}=A_{2}=\frac{T_{S} F_{m n}^{\text {shaker }}}{2 M_{m n} \omega_{m n}^{2} \xi_{m n}} \\
A_{3}=\frac{F_{m n}^{\text {shaker }}}{2 M_{m n} \omega_{m n}^{2} \xi_{m n}}
\end{gathered}
$$

The dis placements field of the plate be ingnow determined, one has to obtain the displacement field in the SHM transducer.

A first hypothesis is to consider a plane stress state for the energy harvester. In that case, the thickness of the energy harvester being small co mpared to its in-plane dimension, it is assumed that the stresses within the thickness are smaller than the in-plane stresses and consequently negligible. In that case, $S_{33}^{P}=0$ and $S_{11}^{P}, S_{22}^{P}$ are not varying within the thickness. In this configuration, only $u_{1}^{P}$ and $u_{2}^{P}$, the tangential displacements, have to be determined and one has equation (18). This model is referred as a 2 dimensions (2D) model.

$$
\begin{aligned}
& u_{1}^{P}\left(x_{1}, x_{2}\right)=u_{1}^{S}\left(x_{1}, x_{2}\right) \\
& u_{2}^{P}\left(x_{1}, x_{2}\right)=u_{2}^{S}\left(x_{1}, x_{2}\right)
\end{aligned}
$$

A second configuration has been developed. It correspondsto a 3D configuration, where all directions are taken into account. The SHM transducer being thin, the influence of the thickness on the tangential components $u_{1}^{P}$ and $u_{2}^{P}$ remains negligible. However, the vertical displacement is not necessarily constant along the thickness as shown by $[27,28]$.

$\mathrm{u}_{1}^{\mathrm{S}}$ and $\mathrm{u}_{2}^{\mathrm{S}}$ applied to the energy harvester will generate a variation of the vertical component of displacement $u_{3}^{p}$. To determine the average displacement variation, represented by the tilde symbol, equation (19) is used.

$$
\widetilde{S_{33}^{P}}=\frac{s_{13}^{E}\left(s_{12}^{E}-s_{11}^{E}\right)}{\left(s_{12}^{E}\right)^{2}-\left(s_{11}^{E}\right)^{2}}\left(\frac{\widetilde{S_{11}^{S}}}{2}+\frac{\widetilde{S_{22}^{S}}}{2}\right)=\frac{\Delta \widetilde{u_{3}^{P}}}{T_{P}}
$$

The displacement field experienced by the SHM energy harvester is subsequently obtained by summing the displacement components applied at the structure/transducer interface and the vertical displacement within the thickness as shown in equation (20). In section 5, this model will be tested and verified using a FEM baseline model.

$$
\begin{gathered}
u_{1}^{P}\left(x_{1}, x_{2}, x_{3}\right)=u_{1}^{S}\left(x_{1}, x_{2}\right)=A_{1} \frac{\partial \phi\left(x_{1}, x_{2}\right)}{\partial x_{1}} \\
u_{2}^{P}\left(x_{1}, x_{2}, x_{3}\right)=u_{2}^{S}\left(x_{1}, x_{2}\right)=A_{2} \frac{\partial \phi\left(x_{1}, x_{2}\right)}{\partial x_{2}} \\
u_{3}^{P}\left(x_{1}, x_{2}, x_{3}\right)=u_{3}^{S}\left(x_{1}, x_{2}\right)+\frac{\widehat{\Delta u_{3}^{P}}\left(x_{1}, x_{2}\right)}{T_{P}} \cdot x_{3}= \\
\left(A_{3}+\frac{\widetilde{\Delta u_{3}^{P}}}{T_{P}}, x_{3}\right) \phi\left(x_{1}, x_{2}\right)
\end{gathered}
$$

\section{Terminal Electric Load}

In order to evaluate the power provided by the piezoelectric SHM energy harvester, a terminal electric load represented by a pure resistor noted $R_{L}$ has been incorporated,[5].

The SHM energy harvester generates an AC voltage while classical electronic equipments need a stabilized DC voltage. Consequently, a full wave rectifier bridge is required. A capacitance may also be used to smooth the rectified voltage leading to a classical circuit for rectifying and s moothing an alternating voltage[8], [30], as represented in Figure 6. $\operatorname{In}[14]$, the authors used a Linear Technology component integrating a full-wave rectifier as well as a storage capacitance.

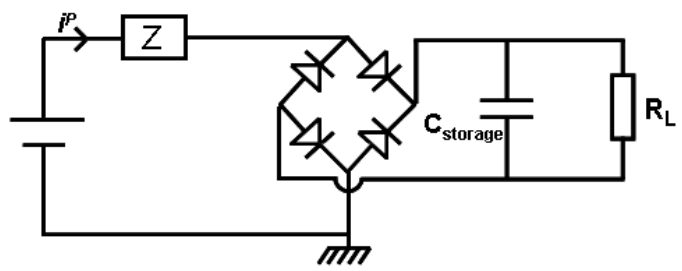

Figure 6. SHM energy harvester and terminal electric load

In the following modelling, it has been assumed that the power is directly transmitted to the resistive load. As a result, the diodes and the smoothing capacitance have not been represented. Only the efficiency of this rectifier bridge, $\eta=\frac{P_{A C}}{P_{D C}}$ with $\mathrm{P}_{\mathrm{DC}}$ the DC output power and $\mathrm{P}_{\mathrm{AC}}$ the $\mathrm{AC}$ input power, has been taken into account. Consequently, the Bond Graph model of the complete energy harvesting process is readily obtained as shown in Figure 7.

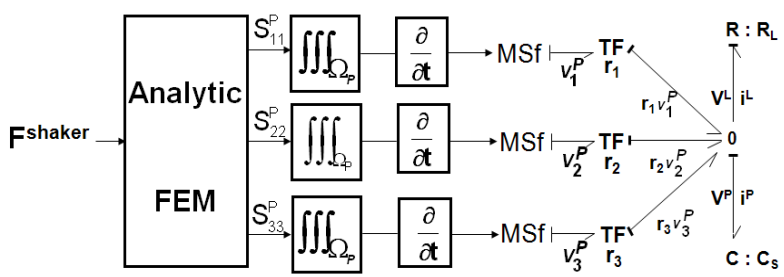

Figure 7. Complete Bond Graph model of the SHM energy harvester

At this stage, the energy harvesting process is completely modelled using the Bond Graph approach.

Running this model provides the harvested power for each given mechanical excitation.

\section{Results and Models Validation}


In this section, the Bond Graph submodels presented in the previous parts are computed using the software 20-sim 4.1 .

A FEM model of the set-up has been built to check the accuracy of the models. So me part of the modelling aspects have been already checked in[14] by comparing the FEM model with experimental results.

\subsection{Structural Mechanical Vibration}

The aluminiu $\mathrm{m}$ late $\left(\mathrm{L}_{\mathrm{S}}=500 \mathrm{~mm}, \mathrm{~W}_{\mathrm{S}}=300 \mathrm{~mm}, \mathrm{~T}_{\mathrm{S}}=3 \mathrm{~mm}\right)$ simply supported on its edges and instrumented with the SHM piezoelectric energy harvester has been modelled by FEM using the software ComsolMultiphysics 4.2. The SHM energy harvester is a square shape PZT Pz 27 from Piezoceramics A/S. Its dimensions are $L_{P}=25 \mathrm{~mm}$, $\mathrm{W}_{\mathrm{P}}=25 \mathrm{~mm}, \mathrm{~T}_{\mathrm{P}}=0.5 \mathrm{~mm}$. The adding of the piezoelectric SHM energy harvester is assumed to not modify the deformation of the plate. However, the presence of the energy harvester implied the need of a refinement of the meshing in the vicinity of the bonding location as shown in Figure 8. In order to improve the efficiency of the computing, the meshing size has been increased in others areas to counterbalance the previous refinement. The total meshing is composed of 14520 tetrahedral elements. The boundaries conditions are the four simply supported edges, modelled by constraining the vertical displacements of these edges. A modal analysis has been conducted to determine the plate's natural frequencies. Then, at each natural frequency, an harmonic analysis has been performed to obtain the displacement field within the SHM energy harvester.

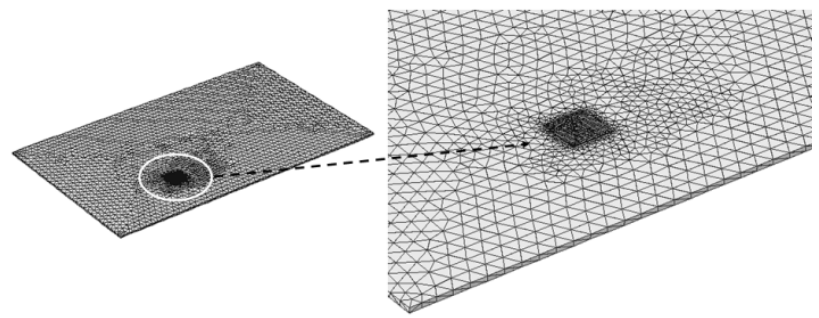

Figure 8. Meshing of the plate instrumented with a SHM energy harvester

The resonance frequencies correspond between the FEM simulation and the analytical modelling. The displacement fields obtained from the analytical plate theory have been also compared with the displacements obtained by the FEM simulation for the first ten natural modes of the structure.As an example, Figures 9 and 10 present the total displacement obtained from the plate theory, i.e. the sum of in-plane and out-of-plane components of the plate at $441 \mathrm{~Hz}$ and $550 \mathrm{~Hz}$, respectively. The theoretical results are close to the results obtained using the FEM model, with, for every case, less than $5 \%$ of difference.

The modelling of the mechanical vibrations using plate theory provided accurate results compared to the FEM simulation, which allowed the validation of the models of the subsystem referred as "Structural mechanical vibrations". Moreover, the SHM energy harvester was not taken into account in the plate theory equations but was integrated in the FEM model. No difference in terms of displacement around the SHM energy harvester area has been noticed. This confirms that the presence of the SHM energy harvester does not modify significantly the displacement field of the structure at these low frequencies.

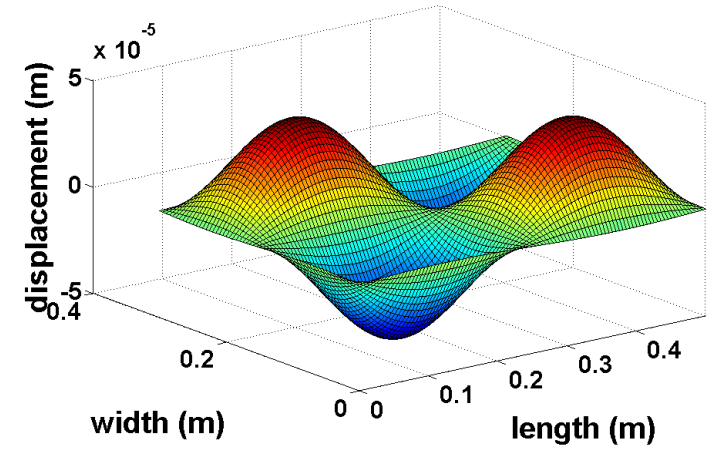

Figure 9. Analytical total plate's displacement at $441 \mathrm{~Hz}$

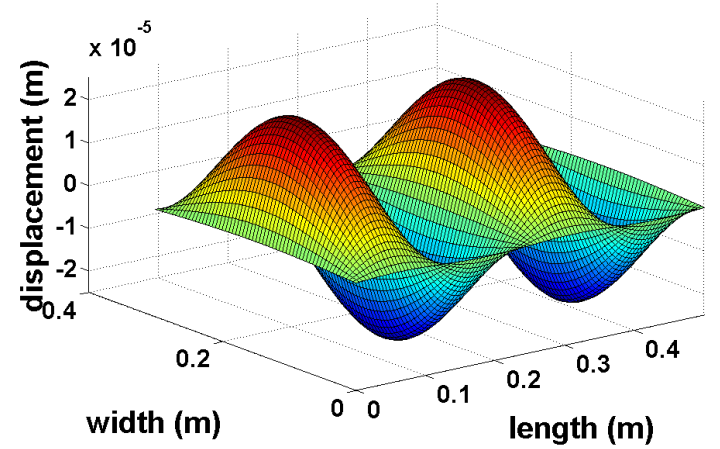

Figure 10. Analytical total plate's displacement at $550 \mathrm{~Hz}$

\subsection{Piezoelectric Electro-Mechanical Conversion}

This electro-mechanical conversion and the subsequent voltage across the piezoelectric energy harvester static capacitance are obtained running the Bond Graph model for the two configurations used in this paper i.e. the $2 \mathrm{D}$ plane stress case and the $3 \mathrm{D}$ configuration. As explained in section 2 , the voltage response corresponds to an open circuit configuration.

The first configuration tested is the plane-stress case. In that case, only the first two branches of the Bond Graph model are considered. This formulation has been tested for the ten natural modes of the plate determined in section 3 and compared with the FEM voltage response. The results provided by the Bond Graph model are presented in Figure 11. One can note a major difference between the plane-stress voltage response and the FEM response taken as a baseline. To reduce this difference, and following the work of A li [16], the influence of the SHM energy harvester thickness has been evaluated.

The second configuration treats the $3 \mathrm{D}$ response of the SHM energy harvester. With the displacements determined analytically as presented in section 3 , and the corresponding Bond Graph parameters (equation (7) to equation (10)), the analytical voltage responses obtained from the Bond Graph 
model have been computed and are also presented in Figure 11.

The voltage response taking into account the three directions of the SHM energy harvester, i.e. the $S_{11}^{P}, S_{22}^{P}$ and $S_{33}^{P}$ components, is close to the baseline FEM model and brings indication of the level of voltage one can expect from a given mechanical excitation at a given frequency. These close voltage responses validate the $3 \mathrm{D}$ model that has been developed. Moreover, this shows that the variation of displacement within the thickness has been correctly modelled with the approximation done in equations (19) and (20). Moreover, this analytical approach provides the required information to evaluate the energy harvesting capabilities of the system. This model can be used to determine what frequencies should be chosen to harvest energy at a promising level and wh ich frequencies should not been chosen for energy harvesting purpose, here $344 \mathrm{~Hz}$ and $588 \mathrm{~Hz}$. It also shows the importance of the vertical contribution in the voltage response since taking into account the vertical component of displacement leads to more accurate results.

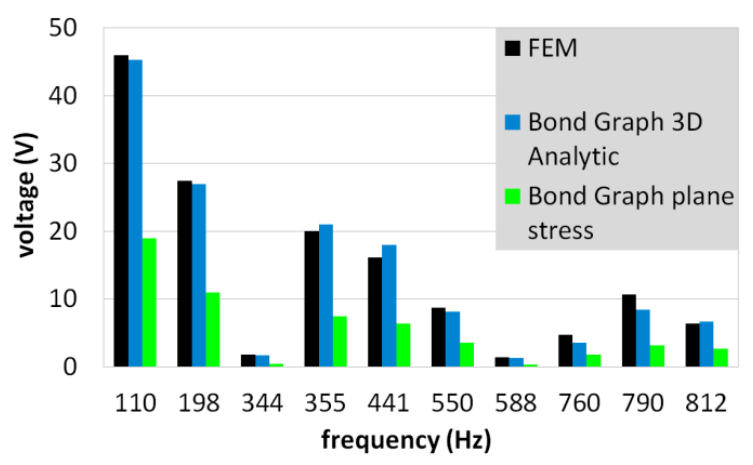

Figure 11. Open circuit voltage response $\mathrm{V}^{\mathrm{P}}$ of the SHM energy harvester

\subsection{Terminal Electric Load}

One of the major features of the Bond Graph is also the possibility to evaluate and monitor the power harvested and transmitted to the terminal electric load. With equation (5) or with the previous FEM model, the voltage response of the SHM energy harvester is readily determined. In this case, one considered the SHM energy harvester to be an open circuit. However, in this configuration, there is no power transfer. Therefore, to determine how much power this SHM energy harvester is able to provide, a load had to be taken into account. The Bond Graph approach allows to rapidly and easily determine this power transfer as a load can be readily added to the SHM energy harvester.

Running the complete model, the level of power harvested is determined for various values of resistive load. This provides the maximum of power that can be harvested as well as the optimum resistive load as presented in Figure 12. This simulation can be conducted for all the previously determined natural modes of the vibrating structure as well as for various locations of the SHM energy harvester. One can note that, for this specific configuration, the optimum load seems to decrease with the frequency increasing. The maximu m of power also depends of the level of deformation at the different natural frequencies. As an example, at $110 \mathrm{~Hz}$ and $355 \mathrm{~Hz}$, the maximum power that can be harvested are $6.8 \mathrm{~mW}$ and $3.5 \mathrm{~mW}$, respectively.

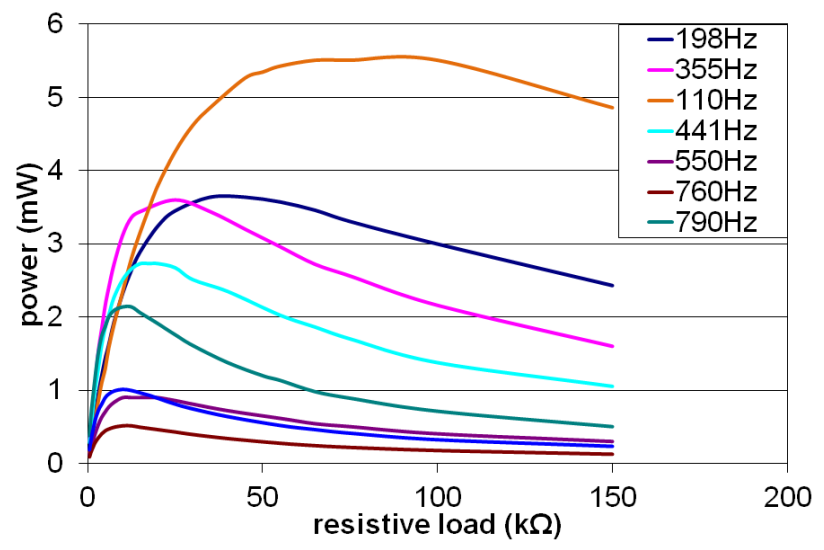

Figure 12. Optimum resistive load and maximum power for the structure natural modes

\section{Conclusions}

In this paper, a novel technical solution has been proposed to obtain a self-powered SHM system for aeronautical applications. This system has a double functionality since it carries out, with a single piezoelectric SHM transducer both the SHM tasks and the vibrational energy harvesting.

The energy harvesting process of this autonomous SHM system has been completely modelled using an innovative Bond Graph approach. The Bond Graph approach has been chosen since it is particularly well suited to study the power and energy transfer within a multiphysics system. The three steps of the energy harvesting process have been modelled. First, the structural mechanical vibrations of the plate have been determined. Then, the piezoelectric electro mechanical conversion submodel provided the energy harvester voltage response to the displacements applied by the vibrating structure. Finally, thanks to the Bond Graph capabilities, the power harvested and transferred to a resistive load has been evaluated. A baseline FEM model has been used to verify the accuracy of the mechanical vibration modelling as well as the SHM energy harvester voltage response. Good correlations have been found for both submodels.

With this complete model, the full energy harvesting process has been represented analytically. One can know, for a natural mode of the plate and for a mechanical excitation, the level of power that can be harvested by a SHM piezoelectric energy harvester. This modelling can lead to numerous applications like comparison between different types of piezoelectric materials, different shapes of energy harvesters and different kinds of terminal load. One can also determine what frequencies are potentially supposed to provide enough mechanical energy to be harvested. This model can consequently be used to optimize the location of the SHM energy harvester and the material to be used. 
The future objectives are to focus on other kinds of mechanical vibrations, not only at the structure resonant frequencies. Other energy harvester shapes will be tested. The work on the loading circuit will be continued to improve the power harvested level.

\section{Nomenclature}

- $\Omega^{\mathrm{P}}$ : volume of the piezoelectric transducer,

- $\Gamma^{\mathrm{P}}$ : surface of the piezoelectric transducer,

- $\mathrm{L}_{\mathrm{P}}=\mathrm{L}_{\mathrm{M}}-\mathrm{L}_{\mathrm{m}}$ : length of the piezoelectric transducer,

- $\mathrm{W}_{\mathrm{P}}=\mathrm{W}_{\mathrm{M}}-\mathrm{W}_{\mathrm{m}}$ : width of the pie zoelectric transducer,

- $\mathrm{T}_{\mathrm{P}}$ : thic kness of the piezoelectric transducer,

- $\mathrm{L}_{S}$ : length of the mechanical structure,

- $\mathrm{W}_{\mathrm{s}}$ : width of the mechanical structure,

- $\mathrm{T}_{\mathrm{S}}$ : thickness of the mechanical structure,

- $\mathrm{F}^{\text {shaker }}$ : input excitation force applied on the structure to generate vibrations,

- $\mathrm{u}_{\mathrm{i}}^{\mathrm{S}}$ : resulting displacement field at the top surface of the structure,

- $\mathrm{F}_{\mathrm{i}}^{\mathrm{P}}$ : resulting force components applied to the piezoelectric transducer,

- $\mathrm{v}_{\mathrm{i}}^{\mathrm{P}}$ : corresponding velocity components applied to the piezoelectric transducer,

- $u_{i}^{\mathrm{P}}$ : resulting displacement field applied to the piezoelectric transducer within its volume,

- $\mathrm{V}^{\mathrm{P}}$ : voltage exhibited by the piezoelectric transducer,

- $\mathrm{i}^{\mathrm{P}}$ : corresponding current flowed by the piezoelectric transducer,

- $\mathrm{V}^{\mathrm{L}}$ : voltage applied to a resistive load,

- $\mathrm{i}^{\mathrm{L}}$ : corresponding current flowed to the resistive load,

- $\mathrm{E}^{\mathrm{P}}$ : electrical field,

- $\mathrm{D}^{\mathrm{P}}$ : electrical displacement,

- $\mathrm{T}^{\mathrm{P}}$ : mechanical stress applied to the piezoelectric transducer,

- $\mathrm{S}^{\mathrm{P}}$ : mechanical strain,

- h: piezoelectric matrix,

- $\mathrm{C}^{\mathrm{D}}$ : stiffness matrix measured with open circuit,

- $\beta^{\mathrm{S}}$ :impermittivity matrix at constant strains,

- $\varepsilon^{\mathrm{T}}$ : permittivity matrix at constant stress,

- $S_{\mathrm{ij}}^{\mathrm{p}}$ : mechanical strain components,

- $\mathrm{C}_{\mathrm{S}}$ : static capacitance of the piezoelectric transducer,

- $\mathrm{r}_{\mathrm{i}}$ : TF elements modelling the electromechanical conversion in the $\mathrm{i}$-d irection,

- $\mathrm{x}_{1,0}, \mathrm{x}_{2,0}$ : coordinates of the force application,

- $\rho$ : density of the mechanical structure (alu minium),

- E : Young modulus of the mechanical structure (alu minium),

- $v$ : Poisson coefficient of the mechanical structure (alu minium),

- vouble Lap lacian operator

$$
\nabla^{4}=\Delta^{2}=\frac{\partial^{4}}{\partial x_{1}^{4}}+2 \frac{\partial^{4}}{\partial x_{1}^{2} \partial x_{2}^{2}}+\frac{\partial^{4}}{\partial x_{2}^{4}}
$$

- Bending stiffness:

$$
\mathrm{D}=\frac{\mathrm{E}\left(\mathrm{T}_{\mathrm{S}}\right)^{3}}{12(1-v)}
$$

- Natural pulsation:

$$
\omega_{\mathrm{mn}}=\sqrt{\frac{\mathrm{K}_{\mathrm{mn}}}{\mathrm{M}_{\mathrm{mn}}}}=\sqrt{\frac{\mathrm{D}}{\rho \mathrm{T}_{\mathrm{S}}}}\left[\left(\frac{\mathrm{m} \pi}{\mathrm{L}_{\mathrm{S}}}\right)^{2}+\left(\frac{\mathrm{n} \pi}{\mathrm{W}_{\mathrm{S}}}\right)^{2}\right]
$$

- Modal deformation:

$$
\phi_{\mathrm{mn}}\left(\mathrm{x}_{1}, \mathrm{x}_{2}\right)=\sin \left(\frac{\mathrm{m} \pi}{\mathrm{L}_{\mathrm{S}}} \mathrm{x}_{1}\right) \sin \left(\frac{\mathrm{n} \pi}{\mathrm{W}_{\mathrm{S}}} \mathrm{x}_{2}\right)
$$

- Damping factor: $\varepsilon_{m n}$, fixed at 0.01 for a standard mechanical system

- Generalized mass:

$$
\mathrm{M}_{\mathrm{mn}}=\rho \mathrm{T}_{\mathrm{S}} \int_{0}^{\mathrm{L}_{\mathrm{S}}} \int_{0}^{\mathrm{W}_{\mathrm{S}}} \phi_{\mathrm{mn}}^{2}\left(\mathrm{x}_{1}, \mathrm{x}_{2}\right) \mathrm{dx}_{1} \mathrm{dx}_{2}=\frac{\rho \mathrm{T}_{\mathrm{S}} \mathrm{L}_{\mathrm{S}} \mathrm{W}_{\mathrm{S}}}{4}
$$

- Modal force:

$$
\begin{aligned}
& \mathrm{F}_{\mathrm{mn}}^{\text {shaker }}(\mathrm{t})=\int_{0}^{\mathrm{LS}_{\mathrm{S}}} \int_{0}^{\mathrm{W}_{\mathrm{S}}} \mathrm{F}^{\text {shaker }}(\mathrm{t}) \delta\left(\mathrm{x}_{1}-\mathrm{x}_{1,0}\right) \\
& \delta\left(\mathrm{x}_{2}-\mathrm{x}_{2,0}\right) \phi_{\mathrm{mn}}\left(\mathrm{x}_{1}, \mathrm{x}_{2}\right) \mathrm{dx}_{1} \mathrm{dx}_{2} \\
& =\mathrm{F}^{\text {shaker }}(\mathrm{t}) \sin \left(\frac{\mathrm{m} \pi \mathrm{x}_{1,0}}{\mathrm{~L}_{\mathrm{S}}}\right) \sin \left(\frac{\mathrm{n} \pi \mathrm{x}_{1,0}}{\mathrm{~W}_{\mathrm{S}}}\right)
\end{aligned}
$$

- Efficiency of the rectifier bridge

$$
\eta=\frac{P_{A C}}{P_{D C}}
$$

- $P_{\mathrm{DC}}$ DC output power,

- $\mathrm{P}_{\mathrm{AC}}$ : AC input power.

\section{REFERENCES}

[1] C.O. Mathuna, T. O'Donnell, R.V. Martinez-Catala,J. Rohan and B. O'Flynn, "Energy scavenging for long-term deployable wireless sensor networks", Elsevier, Talenta, vol.75, no.3, pp. 613-623, 2007.

[2] A. Harb, "Energy harvesting: State-of-the-art", Elsevier, Renewable Energy, vol.36, no.10, pp. 2641-2654, 2011.

[3] G. Park, C.R. Farrar, M.D. Todd, W. Hodgkiss and T. Rosing, "Energy Harvesting for Structural Health Monitoring Sensor Networks", Los Alamos Laboratory, LA-14314-MS. 2007

[4] D. Samson, M. Kluge, Th. Becker and U. Schmid,"Energy Harvesting for Autonomous Wireless Sensor Nodes in Aircraft", Eurosensor XXIV Conference, Elsevier, Procedia Engineering,vol.5, pp. 1160-1163, 2010.

[5] S. Roundy, P.K. Wright and J. Rabaey, "A study of low level vibrations as a power source for wireless sensor nodes", Elsevier, Computer Communications, vol.26, no.11,pp. 1131-1144,2003.

[6] S. Roundy and P.K. Wright, "A piezoelectric vibration based generator for wireless electronics", IOP science, Smart Materials and Structures, vol.13, no.5, pp. 1131-1142, 2004.

[7] E. Lefeuvre, A. Badel, C. Richard, L. Petit and D. Guyomar, "A comparison between several vibration-powered piezoelectric generators for standalone systems", Elsevier, Sensors and Actuators A: Physical, vol.126, no.2, pp. 405-416, 2006. 
[8] M. Lallart and D. Guyomar,"An optimized self-powered switching circuit for non-linear energy harvesting with low voltage output", IOP science, Smart Materials and Structures, vol.17, no.3, 2008

[9] M. Lallart, C. Richard, L. Garbuio, L. Petit and D Guyomar,"High efficiency, wide load bandwidth piezoelectric energy scavenging by a hybrid nonlinear approach", Elsevier, Sensors and Actuators A: Physical, vol.165, no.2, pp. 294-302, 2011.

[10] K.M. Farinholt, N. Miller, W. Sifuentes, J. MacDonald, G. Park and C.R. Farrar,"Energy Harvesting and Wireless Energy Transmission for Embedded SHM Sensor Nodes", SAGE Publications, Structural Health Monitoring, vol.9, no.3, pp. 269-280, 2010.

[11] M. Ferrari, V. Ferrari, M. Guizzetti, D. Marioli and A. Taroni,"Piezoelectric multifrequency energy converter for power harvesting in autonomous microsystems", Elsevier, Sensors and Actuators A: Physical, vol.142, no.1, pp. 329-335, 2008

[12] H. Sodano, E.A. Magliula, G. Park and D.J. Inman, "Electric Power Generation using Piezoelectric Devices", in Proceedings of the Thirteenth International Conference on Adaptive Structures and Technologies, Potsdam, Germany, 2003.

[13] T. Sainthuile, C. Delebarre, S. Grondel and C.A.Paget,"Vibrational power harvesting for wireless PZT-based SHM applications", in Proceedings of the 5th European Workshop on Structural Health Monitoring, Sorrento, Italy, pp. 679-684, 2010

[14] C. Delebarre, T. Sainthuile, S. Grondel and C.A. Paget, "Power harvesting capabilities of SHM ultrasonic sensors", Smart Material Research, vol.2012, 2012.

[15] T. Sainthuile, C. Delebarre, S. Grondel and C.A.Paget, "Bond Graph Model of a thin SHM Piezoelectric Energy Harvester", in Proceedings of the 8th International Workshop on Structural Health Monitoring, Stanford, CA, USA, pp. 618-625, 2011

[16] F.L. Di Scalea, H. Matt and I. Bartoli,"The response of rectangular piezoelectric sensors to Rayleigh and Lamb ultrasonic waves", ASA publications, J. Acoust. Soc. Am., vol.121, no.1, pp. 175-187,2007.

[17] A. Raghavanand E.S. Cesnik, "Finite-dimensional piezoelectric transducer modeling for guided wave bases structural health monitoring", IOP science, Smart Materials and Structures, vol.14, no.6,pp. 1448-1461, 2005

[18] B. Chapuis, “Contrôle Santé Intégré par méthodeultrasonore des réparations composites colléessur des structures métalliques", Ph.D. Dissertation, Université Denis Diderot Paris VII, Paris, France, 2010.
[19] D.C. Karnopp, D.L. Margolis and R.C. Rosenberg,"System Dynamics: Modeling and Simulation of Mechatronic Systems”, John Wiley \& Sons Inc.,USA, 2006.

[20] M. Vergé and D. Jaume, "ModélisationStructurée des systèmes avec les Bond Graphs", Editions Technip, France, 2004.

[21] V.G.M. Annamdas,"Facts of Piezo Impedance Technique in Crack Propagation Studies for an Engineering Structure", Scientific \& Academic Publishing, International Journal of Aerospace Sciences, vol.1, no.2, pp. 8-15, 2012.

[22] S. Grondel, C.A. Paget, C. Delebarre, J. Assaad and K. Levin, "Design of optimal configuration for generating A0 Lamb mode in a composite plate using piezoceramictransducers",A SApublications,J. Acoust. Soc. Am, vol.112, no.1, pp. 84-90, 2002.

[23] C.A. Paget, K. Atherton and E.W. O'Brien, "Triangulation algorithm for damage location in aeronautical composite structures", in Proceedings of the 4th International Workshop on Structural Health Monitoring, Stanford, CA, USA, pp. $363-370,2003$.

[24] C. A.Paget, K. Tiplady, M. Kluge, T. Becker and J. Schalk, "Feasibility study on wireless impact damage assessment system for thick aeronautical composites", in Proceedings of the 5th European Workshop on Structural Health Monitoring, Sorrento, Italy, pp. 125-130, 2010.

[25] F. Benmeddour, S. Grondel, J. Assaad and E. Moulin, "Study of the fundamental Lamb modes interaction with asymetrical discontinuities", Elsevier, NDT \& E International, vol.41, no.5, pp. 330-340, 2008.

[26] F. Benmeddour, S. Grondel, J. Assaad and E. Moulin, "Experimental study of the A0 and S0 Lamb waves interaction with symmetrical notches", Elsevier, Ultrasonics, vol.49, no.2, 202-205,2009.

[27] R. Ali, D.R. Mahapatra and S. Gopalakrishnan, "An analytical model of constrained piezoelectric thin film sensors", Elsevier, Sensors and Actuators A: Physical, vol.116, no.3, pp. 424-437,2004.

[28] R. Ali, "Time-space analysis of a surface-bonded piezoelectric film's response with Lamb wave for diagnostics", Elsevier, Sensors and Actuators A: Physical, vol.161, no.1-2, pp. 12-22, 2010.

[29] J.L. Guyader, "Vibrations des milieuxcontinus", Hermès Science Publications, Paris,2002.

[30] Y.C. Shu and I.C. Lien, "Efficiency of energy conversion for a piezoelectric power harvesting system", IOP science, J. Micromech. Microeng vol.16, no.11, pp. 2429-2438, 2006. 